\title{
A validation of a new measure of activity in psychosis
}

Suzanne Jolley ${ }^{1 *}$, Philippa A. Garety ${ }^{1}$, Lynn Ellett ${ }^{1}$, Elizabeth Kuipers ${ }^{1}$, Daniel Freeman $^{1}$, Paul E. Bebbington ${ }^{2}$, David G. Fowler ${ }^{3}$, Graham Dunn ${ }^{4}$

${ }^{1}$ King's College London, Institute of Psychiatry, Department of Psychology, University of London, UK.

${ }^{2}$ Department of Mental Health Sciences, Royal Free and University College Medical School, UCL, University of London, UK.

${ }^{3}$ School of Medicine, Health Policy and Practice, University of East Anglia, UK.

${ }^{4}$ Biostatistics Group, School of Epidemiology \& Health Sciences, University of Manchester, UK.

*Correspondence to Suzanne Jolley, Department of Psychology, PO Box 77, Institute of Psychiatry, Denmark Hill, London, SE5 8AF, UK

e-mail: s.jolley@iop.kcl.ac.uk

Telephone: (0044) 0207848 5028; Fax: (0044) 02078485006 


\begin{abstract}
Despite demonstrated relationships between activity and clinical change, we lack effective measures of time use in psychosis. Existing time budget measures of activity are demanding to complete, and thus unsuited to routine clinical use as measures of change. Less burdensome 'check-box' measures are prone to bias and omission in the activities selected. We recently devised a simplified time budget measure of activity in psychosis which was piloted on a small sample (Jolley, Garety, Dunn, White, Aitken, Challacombe, Griggs, Wallace, \& Craig, in press). This study is a larger scale validation. 276 participants with a recent relapse of non-affective psychosis completed the new time budget, together with an established measure of global social functioning, measures of positive and negative psychotic symptoms, and positive symptom distress. The time budget measure showed moderate correlations with other indices of social functioning and activity. Results demonstrate relationships between activity levels and psychotic symptomatology, both positive and negative. Positive symptom distress was more strongly associated than symptom severity with activity levels. We conclude that the time budget measure can be used as an indicator of social functioning, with potential as a measure of therapeutic change. We are currently investigating its sensitivity in this context.
\end{abstract}

Keywords: schizophrenia, psychosis, activity, social functioning, time budget 


\section{Introduction}

\subsection{Background}

Deterioration in social functioning is a key symptom of schizophrenia, and the drive to develop measurements of functioning and to understanding the causes of the deterioration in functioning has generated a large body of research. While many measures of social functioning appear to relate more to cognitive deficits than to psychotic symptoms (Green et al., 2000), measures of activity, one component of social functioning, have been reliably associated with symptomatic improvement.

Wing and Brown (1970), in their highly influential follow-up of long-stay hospital patients, demonstrated that reduced time spent doing nothing and increased social contact were the most reliable predictors of improvement in positive and negative psychotic symptoms. Olbrich et al. (1993) used a similar approach, but weighted activities according to subjective and objective demand, and similarly found a close relationship between activity and symptomatic status. Purvis et al. (2004) found a time budget diary and an actimeter rating both showed activity increasing as symptomatic recovery took place in an early psychosis group.

Such time budget measures, requiring hour-by-hour, or even more frequent, recording are demanding to complete, particularly for people with psychosis, and are not suited to routine clinical use. While many social functioning measures have been designed with routine clinical use and sensitivity to change in mind (Weismann, 1975; 1981) few of these measure daily activity. An exception is the Social Functioning Scale (SFS) of Birchwood et al. (1990) which includes several subscales assessing activity 
and social contact. The SFS scales comprise lists of possible activities, which respondents tick according to frequency of occurrence. However, while easy to use, and demonstrably sensitive to change (Birchwood and Smith, 1987; Barrowclough and Tarrier, 1990), a check-box approach cannot deliver individualised accounts of activities and is subject to bias in the selection of activities.

\subsection{A new time budget measure}

We have recently devised a simplified time budget measure, with the aim of creating an individualised, culturally non-specific record of activity, which is simple to complete and repeatable in routine clinical use.

The measure consists of a week long diary of activity, in 4 time periods for each day completed retrospectively during a structured interview with participants.

Interviewers probe for activities, degree of independence in activities, and number and nature of social contacts. The aim is to be comprehensive - covering domestic activities, social contacts, work and leisure. The result is a highly individualized record of activity over the week. Each activity period is then rated according to the complexity of activity and the effort required over and above doing nothing: from passive (watching TV) through active but simple (going to a local shop for a single item), to increasingly active and more complex activities (rehabilitative work, more demanding or lengthy social situations).

While a scoring system of this kind is potentially problematic, and our system is biased towards active, rather than reflective ways of spending time, such a bias is present in many measures (in Wing and Brown's categorization, thinking counts as 
doing nothing) and we believe that this is justified in the context of mental health work, where certain activities (particularly those involving getting out, and socializing, or those that are goal-directed) are promoted as more associated with good mental health than others (such as sitting and thinking alone).

A pilot study of the measure (Jolley et al., in press) has demonstrated good inter-rater reliability, the ability to discriminate between more and less active groups, and moderate relationships with the Birchwood Social Functioning Scale (SFS, Birchwood et al., 1990). In line with our hypothesis, relationships were with activity related scales of the SFS, particularly the Withdrawal subscale, but not the Competence subscale.

\subsection{The present study}

This study was designed as a larger scale validation, investigating particularly the relationship between our activity based measure of social functioning, and both positive and negative psychotic symptoms. If the time budget measure is sensitive to clinical change, it would be expected to show relationships with psychotic symptomatology. However, cognitive models of psychosis (e.g. Garety et al., 2001) emphasise that it is not the symptom or psychotic experience per se which causes problems, but the person's appraisal of the experience. Often, these appraisals centre around the experience being external and threatening to the individual, and lead to high levels of distress. The distress about the symptom or experience may then interfere with the person's functioning or activity levels - for example, being too afraid to go out because of persecutory beliefs about people outside. Thus, changes in distress might plausibly be argued to influence activity more than presence or absence 
of symptoms. In addition to examining relationships with symptoms therefore, we also planned to examine the association between activity and positive symptom distress.

Studies investigating relationships between social functioning and symptomatology have not generally investigated symptom distress. Harrow et al. (2004) took a dimensional approach to delusional beliefs and found delusional status rather than dimensions of emotional involvement, conviction or self-monitoring predicted social functioning, but did not include a measure of distress. Other studies, though not investigating distress associated with psychotic symptoms per se, indicate that affect might be relevant to levels of functioning. Wetherell et al. (2003) in a sample of older adults with psychosis found that anxiety is strongly related to levels of functioning. Davis et al. (2004) discussed the role of aspects of hopelessness, and found a contribution of specific aspects of hopelessness to work functioning. Voges and Addington (2005) found a relationship between negative self-statements and social functioning in an early psychosis sample. No study has so far examined symptom distress in relation to activity.

We hypothesised, that our activity based measure of social functioning, designed to be comparable to measures sensitive to clinical improvement, would show relationships with psychotic symptoms, and, following a cognitive model, particularly the associated distress.

\section{Method}




\subsection{Participants}

276 out of 301 individuals recruited for the Psychological Prevention of Relapse in Psychosis (PRP) Trial (ISRCTN83557988) consented to complete the time budget measure and therefore took part in this study. The PRP trial is a UK multi-centre randomised controlled trial of cognitive behaviour therapy and family intervention for psychosis. It is based in four National Health Service Trusts in London and East Anglia. We aimed to recruit a representative sample of individuals with psychosis at the time of relapse in positive symptoms, either from a previously recovered state or from a milder state of persisting symptoms. The inclusion criteria were: a current diagnosis of non-affective psychosis (schizophrenia, schizo-affective psychosis, delusional disorder) (ICD-10, F20, F25 and F22); age 18-65 years; a second or subsequent episode starting not more than 3 months before consent to enter the trial; and at least one distressing positive psychotic symptom at first time of meeting. The exclusion criteria are: primary diagnosis of alcohol or substance dependency, organic syndrome or learning disability; inadequate command of English to engage in psychological therapy; unstable residential arrangements. The PRP trial, including this study, was carried out in accordance with The Code of Ethics of the World Medical Association (Declaration of Helsinki).

\subsection{Measures}

Basic clinical and demographic data were taken from medical notes: age, sex, ethnicity, marital status, living circumstances (with partner or carer), illness length, number of admissions and inpatient status at recruitment. IQ was measured using the Quick Test (Ammons and Ammons, 1962). Negative symptoms were assessed using 
the Scale for the Assessment of Negative Symptoms (SANS; Andreasen, 1983). Total score and subscores were calculated. As the avolition/apathy subscore of the SANS asks directly about engagement in activities it was treated as an additional index of social functioning rather than of negative symptoms in this study, and the total SANS score excluded avolition/apathy. Positive symptoms were measured using the Psychotic Symptoms Rating Scale (PSYRATS; Haddock et al., 1999). Frequency of hallucinations and conviction in delusional beliefs were used as indices of severity of symptomatology, and ratings of intensity of distress for hallucinations and delusions were used to measure distress. In addition to the avolition/apathy subscore on the SANS, the Social and Occupational Functioning Assessment Scale (SOFAS; APA, 1994) was used as a measure of social functioning.

\subsubsection{The time budget measure}

The measure takes the form of a diary over a week, completed during a structured interview, with 4 time blocks for each day rated from 0-4 as below. Where more than one activity is present, the highest scoring activity is rated. There are 28 time blocks for the week, and the total possible score ranges from 0-112. Interviewers start with the preceding day and probe for activities and social contact over each time period, recording this on a diary sheet for later rating. They also check that the week is a typical or average week, and if not, complete the time budget on a different occasion as well to assess an average week.

The rating system is as follows: 
0 - nothing - lying thinking, sleeping, sitting etc.

1 - predominantly passive activity. e.g. watching TV, listening to the radio

2- an independent activity requiring some planning and motivation, but relatively simple or brief. E.g. a walk to the local shops to get cigarettes, tidying room, washingup, preparing a simple meal for oneself.

3 - several 2-rated activities completely filling a time period, sounding 'busy' or a more complex and demanding but unvaried or shorter activity. e.g. a visit involving public transport, or prolonged social contact with others

4 - time period filled with a variety of demanding independent activities requiring significant motivation and planning and with some variation in tasks. E.g. work, a course of study, a trip out requiring organisation

On a small pilot sample (Jolley et al, in press), inter-rater reliability for the measure has been shown to be high (Intraclass correlation: $\mathrm{r}=0.99, \mathrm{p}<0.001$ ), and test-retest reliability good $(\mathrm{r}=0.83, \mathrm{p}<0.001)$. The measure has good face validity, and was able to discriminate between groups identified by their consultant psychiatrist as high or low in activity levels, and between longer duration and early onset groups with psychosis.

For this study, the week prior to interview was used unless the participant had been an inpatient during that time, as this was likely to restrict the person's activity levels. In 
this case, a week preceding admission, but when the person was still unwell was chosen.

\subsection{Statistical Analyses}

All analyses were conducted using SPSS 11.0 for windows (SPSS, 2001). Data approximated a normal distribution and therefore parametric analyses were used. Following preliminary correlational analysis and ANOVA, a linear regression analysis was used to investigate the relationship between demographic variables and activity. A separate linear regression was conducted to examine the relationship between positive psychotic symptoms, distress and activity.

\section{Results}

\subsection{Demographic and clinical characteristics of sample}

The time budget measure was completed by 276 (197M, 79 F) participants of 301 recruited. Of these, 233 had an ICD-10 diagnosis of schizophrenia, 39 of schizoaffective disorder and 4 of delusional disorder. The majority of participants were single $(73 \%)$ and unemployed (80\%). Carers were identified for 37\%. Means or frequencies for other demographic variables for the study sample are shown in table 1.

Insert table 1 around here 


\subsection{Time budget scores}

The mean score on the time budget measure was 50.1 (SD 19.7); with a spread of scores across the range of the instrument (sample range 2-108; possible range 0-112). In terms of activities, this varied from staying in bed doing nothing most of every day to functioning in full-time employment, with partners and children. In general, those scoring lower on the scale spent most of their time engaged in passive activities or doing nothing, with almost no time periods spent in complex activities, while those at the higher end spent almost no time doing nothing, with a higher level of complex activities. Overall, the sample were engaging in predominantly passive (rating 1) and active but simple and brief (rating 2) activities. Mean frequencies per week (highest possible frequency $=28$ ) of each rating for the whole group and for each quartile are shown in table 2.

Insert table 2 around here

\subsection{Convergent validity}

The correlation between time budget scores and SOFAS scores was moderate $-\mathrm{r}=0.5$, $\mathrm{p}=0.001)$. Table 2 also shows the SOFAS scores for each quartile on the time budget; SOFAS scores were higher for the more active quartiles and lower for those doing less. Time budget scores also correlated moderately with the SANS avolition/apathy

subscale $(\mathrm{r}=0.5, \mathrm{p}<0.001, \mathrm{n}=275)$, and differed significantly according to employment status, those in employment $(n=53)$ scoring a mean of 61.7 (SD 24.2) while those not 
working $(n=221)$ had a mean score of 47.5 (SD 17.4): Mann Whitney $U=3757$, $\mathrm{p}<0.001$.

\subsection{Predictors of variance in time budget scores - demographic variables}

Preliminary correlational analysis and ANOVA revealed associations only between IQ and the time budget $(\mathrm{r}=0.16, \mathrm{p}<0.05, \mathrm{n}=223)$. Age, length of illness and number of admissions all showed no significant correlation with time budget score $(|\mathrm{r}|$ 's range from 0.004 to 0.07 , p's from 0.95 to 0.21 ). ANOVA revealed no variation in time budget scores with gender, ethnicity, marital status, living circumstances (with partner

or carer), or inpatient status at recruitment (F's range from 0.49 to 1.74 , p's from 0.69 to 0.19 ). This was confirmed by linear regression with time budget scores as the dependent variable. The model was not significant $(\mathrm{R}=0.22, \mathrm{~F}(10,184)=0.96$, $\mathrm{p}=0.47)$ and only IQ was found to be a predictor of time budget score $(\mathrm{B}=0.15$, $\mathrm{p}=0.04)$. No other demographic variables were significant predictors $(|\mathrm{B}|$ 's range from 0.001 to 0.1 , p's from 0.99 to 0.21 ). As the inclusion of IQ reduced the sample size, the analysis was repeated excluding IQ, and the pattern of results remained the same $(\mathrm{R}=0.15, \mathrm{~F}(9,231)=0.60, \mathrm{p}=0.79 ;|\mathrm{B}|$ 's range from 0.01 to $0.07, \mathrm{p}$ from 0.88 to 0.39$)$.

\subsection{Predictors of time budget scores - clinical variables}

Preliminary correlational analysis revealed small but highly significant associations between the time budget and the symptom measures. These are presented in table 3 . Higher levels of symptoms were associated with lower levels of activity. The total SANS score (excluding the avolition and apathy subscore), total PSYRATS symptom 
severity and total PSYRATS symptoms distress scores were entered into a linear regression with the time budget score as the dependent variable. The model was significant $(r=0.37, F(3,270)=14.5, p<0.001)$ and an adjusted $r$ squared of 0.13 indicated that a small amount of the variance in activity was accounted for. Negative symptoms $(B=-0.25, \mathrm{p}<0.001)$ and distress $(B=-0.15, \mathrm{p}=0.03)$ were significant predictors, while total positive symptom score was not a significant predictor $(\mathrm{B}=-$ $0.11, \mathrm{p}=0.11$ ). The regression was repeated with IQ included as a predictor variable. Results were similar, with IQ just failing to reach significance as a predictor $(\mathrm{r}=0.37$, $\mathrm{F}(4,216)=8.37, \mathrm{p}<0.001$, adjusted $\mathrm{r}$ square $=0.12$ : IQ $\mathrm{B}=0.12, \mathrm{p}=0.06$; distress $\mathrm{B}=-$ $0.17, \mathrm{p}=0.03$; positive symptoms $\mathrm{B}=-0.06, \mathrm{p}=0.46$; negative symptoms $\mathrm{B}=-0.23$, $\mathrm{p}<0.001)$.

Insert table 3 around here

\section{Discussion}

\subsection{Implications of findings}

This study was a validation of a new time budget measure of activity in psychosis against measures of symptoms, distress, and other measures of functioning and activity.

In terms of the activity levels demonstrated by participants, our study illustrates the wide variability in a group with schizophrenia spectrum diagnoses and a relapsing pattern, from almost total inactivity to very high levels of complex activity. Interestingly, activity patterns were not related to demographic variables, or to living 
circumstances, such that those living with somebody were no more or less active than those living alone.

In line with predictions, the time budget measure shows small but highly significant relationships with both negative symptoms and positive symptom distress. As positive symptoms and the associated distress are obviously highly correlated, the failure of positive symptoms to reach significance as a predictor variable should be treated with caution. However, it is clear that symptom distress is at least of equal importance, and warrants further examination in terms of its relationship with social functioning.

In much of the social functioning literature, relationships between functioning and psychotic symptomatology are not found, or are eclipsed by stronger relationships with cognitive deficits (Green et al., 2000). We have not included measures of specific cognitive deficits in this study; however, the Quick Test IQ measure might be thought of as a proxy measure for cognitive deficits, and while this shows a relationship with our activity measure, a contribution is still made by negative symptoms and positive symptom distress.

The relationship between our measure and symptomatology suggests that, like the time budget measures from which it is derived, our measure will show sensitivity to clinical change.

The validity of the new measure as an indicator of social functioning is supported by the relationship with the SOFAS, and by its ability to discriminate between employed and unemployed participants. The high correlations with the avolition/apathy subscale 
of the SANS, a subscale asking particularly about activities and withdrawal, indicates that our measure does indeed tap activity levels and social contact.

\subsection{Limitations and future research}

One limitation of this study is the selective nature of the sample. Participants were drawn from a study examining the reduction and prevention of relapse of positive symptoms of psychosis. Therefore we have specifically chosen people with current positive symptomatology, and it is possible that relationships with activity would be different in an unselected group with psychosis.

It is also of note that most of the variance $(87 \%)$ in the time budget measure is unaccounted for in our model. This is not decreased by adding IQ as a predictor, which might be considered to be a proxy for cognitive impairment. However, the limited contribution of both symptoms and cognitive impairment are not novel findings in the social functioning literature. It is clear from Green et al.'s (2000) review, that although some studies find large amounts of variance in social functioning accounted for by composite measures of cognitive functioning (e.g. the MMSE accounts for $40-50 \%$ of variation in social functioning in studies by Harvey et al., 1998 and Velligan et al., 1997), the pooled correlation coefficients in the metaanalysis of studies using non-composite measures are between 0.2 and $0.4-$ comparable to our own correlations. The relationships we find, therefore, are not dissimilar in magnitude to other studies, but do suggest that a large amount of the variance in activity levels, both in our sample, and in other research, is unaccounted for, and that other potential contributors to poor social functioning in this clinical 
group should be considered. Possible candidates include current employment status (although in our study, post hoc analyses including this accounted for only $2 \%$ more variance than our existing model), and the impact of receiving a diagnosis and entering the mental health system on opportunities and social network.

Finally, we are developing our new measure with the aim of being able to demonstrate changes in an aspect of social functioning - activity - with clinical change. The associations with symptoms and distress reported here are promising, but further research is clearly required to investigate the relationship between symptomatic and affective change and change in activity levels. Our group is currently collecting outcome data for a randomised controlled trial which will be used to investigate the sensitivity of the time budget measure to change. 


\section{Acknowledgement}

This work was supported by a programme grant from the Wellcome Trust (No.

062452). We wish to thank the patients taking part in the trial and the participating teams in the four NHS Trusts. 
Table 1 Demographic and clinical characteristics of participants

\begin{tabular}{|l|c|l|}
\hline Variable & $\mathrm{n}$ & Mean (SD) \\
\hline Age (years) & 276 & $39.5(11.5)$ \\
\hline Length of illness (years) & 269 & $10.8(9.1)$ \\
\hline IQ (Quick Test) & $210^{*}$ & $95.6(12.0)$ \\
\hline SAPS & 276 & $29.8(16.7)$ \\
\hline SANS & 276 & $28.0(18.5)$ \\
\hline Freq. Auditory Hallucinations (PSYRATS) & 276 & $1.3(1.4)$ \\
\hline Intensity distress (AH) & 276 & $1.3(1.5)$ \\
\hline Delusional conviction (PSYRATS) & 276 & $2.7(1.5)$ \\
\hline Intensity distress (Deln) & 276 & $1.9(1.4)$ \\
\hline Beck Anxiety Inventory & 253 & $20.1(14.4)$ \\
\hline Beck Depression Inventory II & 268 & $22.3(13.3)$ \\
\hline Ethnicity & & 216 \\
\hline White & & 25 \\
\hline Black Caribbean & & 6 \\
\hline
\end{tabular}

*The Quick Test was completed only by those whose first language was English 
Table 2: Mean frequencies of 0-4 ratings

\begin{tabular}{|c|c|c|c|c|c|c|c|}
\hline Quartiles & $\begin{array}{c}\text { Time } \\
\text { budget } \\
\text { total (SD) }\end{array}$ & $\begin{array}{c}\text { SOFAS } \\
(\mathrm{SD})\end{array}$ & $\begin{array}{l}\text { Mean } \\
\text { no. } 0 \\
\text { ratings } \\
\text { (SD) }\end{array}$ & $\begin{array}{l}\text { Mean } \\
\text { no. } 1 \\
\text { ratings } \\
\text { (SD) }\end{array}$ & $\begin{array}{c}\text { Mean } \\
\text { no. } 2 \\
\text { Ratings } \\
\text { (SD) }\end{array}$ & $\begin{array}{l}\text { Mean } \\
\text { no. } 3 \\
\text { ratings } \\
\text { (SD) }\end{array}$ & $\begin{array}{l}\text { Mean } \\
\text { no. } 4 \\
\text { ratings } \\
\text { (SD) }\end{array}$ \\
\hline $0-25 \%$ & $\begin{array}{l}28.1 \\
(6.5)\end{array}$ & $\begin{array}{r}41.7 \\
(13.0)\end{array}$ & $\begin{array}{c}8.4 \\
(5.6)\end{array}$ & $\begin{array}{l}12.6 \\
(7.4)\end{array}$ & $\begin{array}{c}5.5 \\
(4.2)\end{array}$ & $\begin{array}{c}1.4 \\
(2.2)\end{array}$ & $\begin{array}{c}0.1 \\
(0.4)\end{array}$ \\
\hline $26-50 \%$ & $\begin{array}{l}42.7 \\
(3.9)\end{array}$ & $\begin{array}{c}50.5 \\
(11.8)\end{array}$ & $\begin{array}{l}2.3 \\
(3.4)\end{array}$ & $\begin{array}{l}12.2 \\
(6.1)\end{array}$ & $\begin{array}{l}10.3 \\
(5.0)\end{array}$ & $\begin{array}{l}3.1 \\
(3.0)\end{array}$ & $\begin{array}{c}0.2 \\
(0.6)\end{array}$ \\
\hline $51-75 \%$ & $\begin{array}{l}55.2 \\
(4.0)\end{array}$ & $\begin{array}{c}54.1 \\
(13.7)\end{array}$ & $\begin{array}{c}1.3 \\
(2.4)\end{array}$ & $\begin{array}{r}6.9 \\
(4.1)\end{array}$ & $\begin{array}{l}11.9 \\
(6.9)\end{array}$ & $\begin{array}{r}6.9 \\
(4.0)\end{array}$ & $\begin{array}{c}1.0 \\
(2.0)\end{array}$ \\
\hline $76-100 \%$ & $\begin{array}{c}78.0 \\
(12.1)\end{array}$ & $\begin{array}{c}62.0 \\
(11.0)\end{array}$ & $\begin{array}{c}0.4 \\
(1.0)\end{array}$ & $\begin{array}{l}2.4 \\
(3.3)\end{array}$ & $\begin{array}{r}6.9 \\
(5.8)\end{array}$ & $\begin{array}{l}11.2 \\
(5.9)\end{array}$ & $\begin{array}{l}7.1 \\
(7.4)\end{array}$ \\
\hline $\begin{array}{l}\text { Total } \\
\text { (276) }\end{array}$ & $\begin{array}{c}50.1 \\
(19.7)\end{array}$ & $\begin{array}{c}51.7 \\
(14.4)\end{array}$ & $\begin{array}{c}3.3 \\
(4.8)\end{array}$ & $\begin{array}{l}8.7 \\
(6.9)\end{array}$ & $\begin{array}{r}8.6 \\
(6.1)\end{array}$ & $\begin{array}{l}5.5 \\
5.5)\end{array}$ & $\begin{array}{r}2.0 \\
(4.7)\end{array}$ \\
\hline
\end{tabular}


Table 3. Correlations between clinical variables and activity

\begin{tabular}{|l|c|c|}
\hline Symptom measure & N & Time budget \\
& & (r) \\
\hline SANS Affective flattening & 276 & $-0.21^{* * *}$ \\
\hline SANS Alogia & 276 & $-0.20^{* * *}$ \\
\hline SANS Anhedonia & 274 & $-0.23^{* * *}$ \\
\hline SANS Inattention & 275 & $-0.20^{* * *}$ \\
\hline SANS Total (excl. avolition/apathy) & 276 & $-0.29^{* * *}$ \\
\hline PSYRATS auditory hallns (AH) & 276 & $-0.17^{* *}$ \\
\hline PSYRATS AH distress intensity & 276 & $-0.19^{* *}$ \\
\hline PSYRATS delusional conviction & 276 & $-0.16^{* *}$ \\
\hline PSYRATS delusional distress intensity & 276 & $-0.21^{* * *}$ \\
\hline Combined PSYRATS symptoms & 276 & $-0.22^{* * *}$ \\
\hline Combined PSYRATS distress & 276 & $-0.26^{* * *}$ \\
\hline
\end{tabular}




\section{References}

Ammons, R.B., Ammons, C.H., 1962. Quick Test. Psychological Test Specialists, Missoula, MT.

Andreasen, N.C., 1983. The Scale for the Assessment of Negative Symptoms (SANS). The University of Iowa, Iowa City, IA.

American Psychiatric Association, 1994. Diagnostic and Statistical Manual of Mental Disorders (DSM IV). APA, Washington, DC.

Barrowclough, C., Tarrier, N., 1990. Social functioning in schizophrenic patients. I. The effects of expressed emotion and family intervention. Soc. Psychiatry Psychiatr. Epidemiol. 25, 125-9.

Birchwood, M.J., Smith, J., 1987. Schizophrenia and the Family, in Orford J. (Ed.), Coping with Disorder in the Family. Croom-Helm, Beckenham.

Birchwood, M., Smith, J., Cochrane, R., Wetton, S., Copestake, S., 1990. The Social Functioning Scale: The development and validation of a new scale of social adjustment for use in family intervention programmes with schizophrenic patients. Br. J. Psychiatry 157, 853-9. 
Davis, L.W., Nees, M.A., Hunter, N.L., Lysaker, P.H., 2004. Hopelessness as a predictor of work functioning among patients with schizophrenia. Psychiatr. Serv. 55, 434-436.

Endicott, J., Spitzer, R.L., Fleiss, J.L., Cohen, J, 1976. The Global Assessment Scale: a procedure for measuring the overall severity of psychiatric disturbance. Arch. Gen. Psychiatry 33, 766-771.

Garety, P.A., Kuipers, E., Fowler, D., Freeman, D., \& Bebbington, P.E., 2001. A cognitive model of the positive symptoms of psychosis. Psychol. Med. 31, 189-195.

Green, M.F., Kern, R.S., Braff, D.L., Mintz, J, 2000. Neurocognitive deficits and functional outcome in schizophrenia: Are we measuring the 'right stuff'? Schizophr. Bull. 26, 119-136.

Haddock, G., McCarron, J., Tarrier, N., \& Faragher, E.B., 1999. Scales to measure dimensions of hallucinations and delusions: the psychotic symptom rating scales (PSYRATS). Psychol. Med. 29, 879-889.

Harrow, M., Herbener, E.S., Shanklin, A., Jobe, T.H., Rattenbury, F., Kaplan, K.J., 2004. Followup of psychotic outpatients: dimensions of delusions and work functioning in schizophrenia. Schizophr. Bull. 30, 147-161.

Harvey, P.D., Howanitz, E., Parrella, M., White, L., Davidson, M., Mohs, R.C., Hoblyn, J., Davis, K.L., 1998. Am. J. Psychiatry 155, 1080-1086. 
Jolley, S., Garety, P., Dunn, G., White, J., Aitken, M., Challacombe, F., Griggs, M., Wallace, M., Craig, T., in press. A pilot validation study of a new measure of activity in psychosis. Soc. Psychiatry Psychiatr. Epidemiol.

Olbrich, R., Voss, E., Mussgay, L., Pfeiffer, H., 1993. A weighted time budget approach for the assessment of cognitive and social activities. Soc. Psychiatry Psychiatr. Epidemiol. 28, 184-8.

Purvis, R.G., Ohlsen, R.I., O’Toole, M.S., Pilowsky, L.S., Brown, R.G., 2004. Realworld functional outcome in schizophrenia: A goal-directed behaviour model. Schizophr. Res. 62 1S, 200.

SPSS (2001). SPSS Base 11 User's Guide. SPSS Inc., Chicago, IL. Velligan, D.I., Mahurin, R.K., Diamond, P.L., Hazelton, B.C., Eckert, S.L., Miller, A.L., 1997. The functional significance of symptomatology and cognitive function in schizophrenia. Schizophr. Res. 25, 21-31.

Voges, M., Addington, J., 2005. The association between social anxiety and social functioning in first episode psychosis. Schizophr. Res. 76, 287-292.

Weissman, M.M., 1975. The assessment of social adjustment. A review of techniques. Arch. Gen. Psychiatry 32, 357-65. 
Weissman, M.M., Sholomskas, D., John, K., 1981. The assessment of social adjustment. An update. Arch. Gen. Psychiatry 38, 1250-8.

Wetherell, J.L., Palmer, B.W., Thorp, S.R., Patterson, T.L., Golshan, S., Jeste, D.V., 2003. Anxiety symptoms and quality of life in middle-aged and older outpatients with schizophrenia and schizoaffective disorder. J. Clin. Psychiatry 64, 1476-1482.

Wing, J.K., Brown, G.W., 1970. Institutionalism and Schizophrenia: A comparative study of three mental health hospitals 1960-1968. Cambridge University Press, London. 